International Electronic Journal of Geometry

Volume 7 No. 1 PP. 4-25 (2014) C IEJG

\title{
A CONSTRUCTION METHOD OF LAGRANGIAN SURFACES IN COMPLEX PSEUDO-EUCLIDEAN PLANE $\mathrm{C}_{1}^{2}$ AND ITS APPLICATIONS
}

\author{
BANG-YEN CHEN
}

Dedicated to Franki Dillen, a great friend and coworker

\begin{abstract}
An important problem in the theory of Lagrangian submanifolds is to find non-trivial examples of Lagrangian submanifolds in complex Euclidean spaces with some given special geometric properties. In this article, we provide a method to construct a large family of Lagrangian surfaces in the complex pseudo-Euclidean plane $\mathbf{C}_{1}^{2}$ by using either Legendre curves in the pseudo-sphere $S_{2}^{3}(1) \subset \mathbf{C}_{1}^{2}$ or Legendre curves in the anti-de Sitter space $H_{1}^{3}(-1) \subset \mathbf{C}_{1}^{2}$. In the first part of this article, we study basic intrinsic and extrinsic geometric properties of the Lagrangian surfaces in $\mathbf{C}_{1}^{2}$ obtained via our construction method. In the second part we completely classify Lagrangian surfaces of constant curvature and minimal Lagrangian surfaces in $\mathbf{C}_{1}^{2}$ obtained from our construction method.
\end{abstract}

\section{INTRODUCTION.}

An immersion $f: M^{n} \rightarrow \tilde{M}_{s}^{n}$ of an $n$-manifold $M^{n}$ into a pseudo-Kaehler $n$ manifold $\tilde{M}_{s}^{n}$ with complex index $s$ is called a Lagrangian immersion if the complex structure $J$ of $\tilde{M}_{s}^{n}$ interchanges each tangent space of $M^{n}$ with its corresponding normal space. The Lagrangian submanifold $M^{n}$ in $\tilde{M}_{s}^{n}$ has (real) index $s$.

Lagrangian submanifolds arise naturally in many physical and geometric situations. For instance, the systems of partial differential equations of Hamilton-Jacobi type lead to the study of Lagrangian submanifolds and foliations in the cotangent bundle. Furthermore, Lagrangian submanifolds are part of a growing list of mathematically rich special geometries that occur naturally in string theory.

An important problem in the theory of Lagrangian submanifolds is to find nontrivial examples of Lagrangian submanifolds with some given special geometric properties. For instance, a method was given in [22] to construct an important family of special Lagrangian submanifolds in $\mathbf{C}^{n}$ with large symmetric groups. Moreover, a method was introduced in [19] to construct Lagrangian submanifolds of constant sectional curvature by utilizing twisted products decompositions of real space forms. In [1, 2], Aiyama introduced a spinor-like representation formula

2000 Mathematics Subject Classification. Primary 53C40, 53D12; Secondary 53C42.

Key words and phrases. Lagrangian immersion, Legendre curve, Lagrangian surface of constant curvature, Hamiltonian minimal Lagrangian surfaces, Lagrangian catenoid. 
which parameterizes immersions through two complex functions $F_{1}, F_{2}$ and a real one (the Lagrangian angle $\beta$ ). I. Castro and the author introduced in [3] a construction method to construct a large family of Lagrangian surfaces in the complex Euclidean plane $\mathbf{C}^{2}$ by using pairs of spherical and hyperbolic curves. Also, the author has completely classified all Lagrangian surfaces of constant curvature in complex space forms in a series of papers ([9]-[14] and [16]). Recently, spherical Lagrangian submanifolds in $\mathbf{C}^{n}$ are completely classified in [18].

A regular curve $z: I \rightarrow S^{3}(1) \subset \mathbf{C}^{2}$ in the ordinary unit hypersphere $S^{3}(1)$ is called Legendre if $\left\langle z^{\prime}(t), i z(t)\right\rangle=0$ holds identically. In [7], the author introduced a method to construct flat Lagrangian submanifolds in $\mathbf{C}^{n}$ by using special Legendre curves in $S^{2 n-1}(1) \subset \mathbf{C}^{n}$. In particular, his result implies that, for any unit speed Legendre curve $z: I \rightarrow S^{3}(1) \subset \mathbf{C}^{2}$ and any real-valued function $p(t)$ defined on an open interval $I$ containing 0 , the map

$$
L(s, t)=s z(t)+\int_{0}^{t} p(t) z^{\prime}(t) d t
$$

defines a flat Lagrangian surface in $\mathbf{C}^{2}$. This construction method was generalized in [15] to construct a much wider class of Lagrangian surfaces in $\mathbf{C}^{2}$.

The main purpose of this article is to extend the construction method of [15] to a method which produces a very large family of Lagrangian surfaces in the complex pseudo-Euclidean plane $\mathbf{C}_{1}^{2}$ using either Legendre curves in the pseudosphere $S_{2}^{3}(1) \subset \mathbf{C}_{1}^{2}$ or Legendre curves in the anti-de Sitter space $H_{1}^{3}(-1) \subset \mathbf{C}_{1}^{2}$. In the first part of this article, we study basic intrinsic and extrinsic properties of Lagrangian surfaces in $\mathbf{C}_{1}^{2}$ obtained via our method. In the second part we completely classify Lagrangian surfaces of constant curvature as well as minimal Lagrangian surfaces in $\mathbf{C}_{1}^{2}$ obtained via the construction method of this article.

\section{Preliminaries.}

Mostly, we follow the notations and definitions from [17]. Throughout this article we denote by $\mathbf{C}_{s}^{n}$ the complex pseudo-Euclidean $n$-space with complex index $s$ endowed with the complex structure $J$ and the indefinite metric given by

$$
g_{0}=-\sum_{j=1}^{s} d z_{j} d \bar{z}_{j}+\sum_{k=s+1}^{n} d z_{k} d \bar{z}_{k}
$$

for $\left(z_{1}, \ldots, z_{n}\right) \in \mathbf{C}_{s}^{n}$.

2.1. Basic definitions, formulas and notations. Assume that $\phi: M \rightarrow \mathbf{C}_{1}^{2}$ is an isometric immersion of a 2-dimensional pseudo-Riemannian manifold $M$ into the complex pseudo-Euclidean plane $\mathbf{C}_{1}^{2}$. We denote the Riemannian connections of $M$ and $\mathbf{C}_{1}^{2}$ by $\nabla$ and $\tilde{\nabla}$, respectively; and by $D$ the connection on the normal bundle of the surface. Let $\langle$,$\rangle denote the inner product on \mathbf{C}_{1}^{2}$ as well as on $M$.

The formulas of Gauss and Weingarten are given respectively by

$$
\begin{aligned}
& \tilde{\nabla}_{X} Y=\nabla_{X} Y+h(X, Y), \\
& \tilde{\nabla}_{X} \xi=-A_{\xi} X+D_{X} \xi
\end{aligned}
$$

for tangent vector fields $X, Y$ and normal vector field $\xi$. The second fundamental form $h$ is related to the shape operator $A_{\xi}$ by

$$
\langle h(X, Y), \xi\rangle=\left\langle A_{\xi} X, Y\right\rangle .
$$


The mean curvature vector $H$ of $M$ in $\mathbf{C}_{1}^{2}$ is defined by $H=\frac{1}{2}$ trace $h$. The length of $H$ is called the mean curvature function.

If $R$ denotes the Riemann curvature tensor of $\nabla$, then the equations of Gauss and Codazzi are given respectively by

$$
\begin{aligned}
& \langle R(X, Y) Z, W\rangle=\langle h(X, W), h(Y, Z)\rangle-\langle h(X, Z), h(Y, W)\rangle, \\
& (\nabla h)(X, Y, Z)=(\nabla h)(Y, X, Z),
\end{aligned}
$$

where $X, Y, Z, W$ are vector fields tangent to $M$ and $\nabla h$ is defined by

$$
(\nabla h)(X, Y, Z)=D_{X} h(Y, Z)-h\left(\nabla_{X} Y, Z\right)-h\left(Y, \nabla_{X} Z\right) .
$$

When $M$ is a Lagrangian surface in $\mathbf{C}_{1}^{2}$, we also have (cf. $[17,20]$ )

$$
\begin{aligned}
& D_{X} J Y=J \nabla_{X} Y, \\
& \langle h(X, Y), J Z\rangle=\langle h(Y, Z), J X\rangle=\langle h(Z, X), J Y\rangle .
\end{aligned}
$$

A vector $v$ in $\mathbf{C}_{1}^{2}$ is called space-like if $v=0$ or $\langle v, v\rangle>0$ and time-like if $\langle v, v\rangle<0$. It is called light-like or null if $\langle v, v\rangle=0$ and $v \neq 0$.

A regular curve $z: I \rightarrow \mathbf{C}_{1}^{2}$ defined on an open interval $I \subset \mathbf{R}$ is called space-like (resp., time-like or null) if $z^{\prime}$ is space-like (resp. time-like or light-like) at each point. A space-like (resp., time-like) curve is said to be of unit speed if $\left\langle z^{\prime}, z^{\prime}\right\rangle=1$ (resp., $\left\langle z^{\prime}, z^{\prime}\right\rangle=-1$ ) holds identically.

2.2. Legendre curves. A curve $z: I \rightarrow \mathbf{C}_{1}^{2}$ is called a Legendre curve if $\left\langle z^{\prime}, i z\right\rangle=0$ holds identically.

If we put

$$
\begin{aligned}
& S_{2}^{3}(1)=\left\{x \in \mathbf{C}_{1}^{2}:\langle x, x\rangle=1\right\}, \\
& H_{1}^{3}(-1)=\left\{x \in \mathbf{C}_{1}^{2}:\langle x, x\rangle=-1\right\},
\end{aligned}
$$

then $S_{2}^{3}(1)$ and $H_{1}^{3}(-1)$ and $\mathcal{L} C$ are called the pseudo sphere, and anti-de Sitter space, respectively.

We need the following lemmas, analogous to [6, Theorem 4.1(a)], for later use.

Lemma 2.1. We have the following:

(a) If $z: I \rightarrow S_{2}^{3}(1) \subset \mathbf{C}_{1}^{2}$ is a unit speed time-like curve in $S_{2}^{3}(1)$ satisfying the following second order differential equation:

$$
z^{\prime \prime}(t)-i \lambda(t) z^{\prime}(t)-z(t)=0
$$

for some nonzero real-valued function $\lambda$, then $z=z(t)$ is a Legendre curve.

(b) Conversely, if $z: I \rightarrow S_{2}^{3}(1) \subset \mathbf{C}^{2}$ is a unit speed time-like Legendre curve, then it satisfies (2.8) for some function $\lambda$.

Proof. Assume that $z: I \rightarrow S_{2}^{3}(1) \subset \mathbf{C}_{1}^{2}$ is a unit speed time-like curve which satisfies (2.8) for some real function $\lambda$, not identically zero. Then, by taking the derivative of $\left\langle z^{\prime}, z\right\rangle=0$ and applying $\langle z, z\rangle=1$ and $\left\langle z^{\prime}, z^{\prime}\right\rangle=-1$, we find

$$
\left\langle z^{\prime \prime}, z\right\rangle=1 \text {. }
$$

Therefore, after taking the inner product of (2.8) with $z$ and after applying (2.9), we have

$$
\lambda\left\langle z^{\prime}, i z\right\rangle=0
$$


Let us put

$$
U=\{x \in I: \lambda(x) \neq 0\} .
$$

If $U=I$, then (2.10) implies that $\left\langle z^{\prime}, i z\right\rangle=0$ holds identically on $I$. Thus $z$ defines a Legendre curve in $S^{3}(1)$ by definition.

Next, suppose that $U \neq I$, then we have $\lambda=0$ on $I-U$. Because $\left\langle z^{\prime}, i z\right\rangle^{\prime}=0$ on $I-U$, the continuity implies that $\left\langle z^{\prime}, i z\right\rangle=0$ identically. Therefore $z$ defines a Legendre curve in $S_{2}^{3}(1)$.

Conversely, suppose that $z$ is a unit speed time-like Legendre curve in $S_{2}^{3}(1)$, then we have $\left\langle z^{\prime}, i z\right\rangle=0$. After taking its derivative of this equation we find

$$
\left\langle z^{\prime \prime}, i z\right\rangle=0
$$

holds identically. Also, it follows from $\left\langle z^{\prime}, z\right\rangle=0$ and $\left\langle z^{\prime}, z^{\prime}\right\rangle=-1$ that

$$
\left\langle z^{\prime \prime}, z\right\rangle=1 \text {. }
$$

On the other hand, since $z, i z, z^{\prime}$ and $i z^{\prime}$ form a pseudo-orthonormal frame field satisfying

$$
\langle z, z\rangle=\langle i z, i z\rangle=1,\left\langle z^{\prime}, z^{\prime}\right\rangle=\left\langle i z^{\prime}, i z^{\prime}\right\rangle=-1,
$$

we may express $z^{\prime \prime}$ as a linear combination of $z, i z, z^{\prime}, i z^{\prime}$. Thus we have

$$
z^{\prime \prime}=\theta z+\eta i z+\mu z^{\prime}+\lambda i z^{\prime}
$$

for some functions $\theta, \eta, \mu, \lambda$. Now, by applying (2.12), (2.13) and (2.14), we find $\theta=1, \eta=\mu=0$. Consequently, we obtain (2.8).

Similarly, we also have the following.

Lemma 2.2. We have the following:

(a) If $z: I \rightarrow H_{1}^{3}(-1) \subset \mathbf{C}_{1}^{2}$ is a unit speed space-like curve in $H_{1}^{3}(-1)$ satisfying

$$
z^{\prime \prime}(t)-i \lambda(t) z^{\prime}(t)-z(t)=0
$$

for some nonzero real-valued function $\lambda$, then $z=z(t)$ is a Legendre curve.

(b) Conversely, if $z: I \rightarrow H_{1}^{3}(-1) \subset \mathbf{C}^{2}$ is a unit speed space-like Legendre curve, then it satisfies (2.15) for some function $\lambda$.

Proof. This can be done in a similar way as Lemma 2.1.

Remark 2.1. It is easy to verify that the function $\lambda$ given in (2.8) (resp., given in $(2.15)$ ) is the curvature function of the unit speed Legendre curve $z$ in $S_{2}^{3}(1)$ (resp., in $\left.H_{1}^{3}(-1)\right)$.

2.3. Another lemma. We also need the following lemma.

Lemma 2.3. Let $u, v$ be any two vectors in the complex pseudo-Euclidean $n$-space $\mathbf{C}_{s}^{n}$ with complex index $s$ and let $a, b$ be any two complex numbers. Then we have

$$
\begin{aligned}
& \langle a u, b v\rangle=\langle a, b\rangle\langle u, v\rangle+\langle i a, b\rangle\langle u, i v\rangle, \\
& \langle a u, i b v\rangle=\langle a, b\rangle\langle u, i v\rangle+\langle a, i b\rangle\langle u, v\rangle,
\end{aligned}
$$

where $\langle a, b\rangle=\operatorname{Re}(a \bar{b})$ denotes the real part of the complex number $a \bar{b}$ with $\bar{b}$ being the complex conjugate of $b$, and $\langle u, v\rangle$ denotes the canonical inner product of $u$ and $v$ in $\mathbf{C}_{s}^{n}$.

Proof. Follows by straightforward computation. 


\section{INTRINSIC PROPERTIES}

Let $\mathbf{C}^{*}$ denote the punched complex line $\mathbf{C}-\{0\}$. A curve $z: I \rightarrow \mathbf{C}_{1}^{2}$ is called regular if $z^{\prime}$ is nowhere zero.

The following result provides a very simple construction method to produce many Lagrangian surfaces in $\mathbf{C}_{1}^{2}$ via Legendre curves.

Proposition 3.1. Let $f: I_{1} \rightarrow \mathbf{C}^{*}$ be a regular curve defined on an open interval $I_{1}$ of the real line and let $z: I_{2} \rightarrow S_{2}^{3}(1) \subset \mathbf{C}_{1}^{2}$ be a unit speed time-like curve defined on an open interval $I_{2}$ containing 0 . Then, for any functions $p: I_{2} \rightarrow \mathbf{C}$, we have the following.

(i) If $z: I_{2} \rightarrow S_{2}^{3}(1) \subset \mathbf{C}_{1}^{2}$ is a Legendre curve, then

$$
L_{f p z}(s, t)=f(s) z(t)-\int_{0}^{t} p(t) z^{\prime}(t) d t
$$

defines a Lagrangian isometric immersion of $M=\left(U_{f z}, g_{f p z}\right)$ into $\mathbf{C}_{1}^{2}$, where

$$
U_{f z}=\left\{(s, t) \in I_{1} \times I_{2}: f(s) \neq p(t)\right\}
$$

and $g_{f p z}$ is the Lorentzian metric given by

$$
g_{f p z}=\left|f^{\prime}(s)\right|^{2} d s^{2}-|f(s)-p(t)|^{2} d t^{2} .
$$

(ii) Conversely, if $f$ contains no circular arcs in $\mathbf{C}^{*}$ and if $L_{f p z}$ is a Lagrangian immersion, then $z: I_{2} \rightarrow S_{2}^{3}(1) \subset \mathbf{C}_{1}^{2}$ is a Legendre curve in $S_{2}^{3}(1)$.

Proof. Let $f: I \rightarrow \mathbf{C}^{*}$ be a regular curve, $z: I_{2} \rightarrow S_{2}^{3}(1) \subset \mathbf{C}_{1}^{2}$ be a unit speed time-like curve, and let $L=L_{f p z}$ be the map defined by (3.1). Then we have

$$
\begin{aligned}
L_{s} & =\frac{\partial L}{\partial s}=f^{\prime}(s) z(t), \\
L_{t} & =\frac{\partial L}{\partial t}=(f(s)-p(t)) z^{\prime}(t) .
\end{aligned}
$$

Since $\left\langle z, z^{\prime}\right\rangle=0$, Lemma 2.1 and (3.4) imply that

$$
\begin{aligned}
& \left\langle L_{s}, L_{s}\right\rangle=\left|f^{\prime}(s)\right|^{2}, \\
& \left\langle L_{s}, L_{t}\right\rangle=\left\langle i f^{\prime}, f-p\right\rangle\left\langle z, i z^{\prime}\right\rangle, \\
& \left\langle L_{t}, L_{t}\right\rangle=-|f-p|^{2} .
\end{aligned}
$$

If $z(t)$ is a Legendre curve in $S_{2}^{3}(1) \subset \mathbf{C}_{1}^{2}$, we have $\langle z, z\rangle=1$. Thus it follows from (3.4) and (3.5) that the induced metric on $U_{f z}$ is given by (3.3). Since $z(t)$ is a Legendre curve, (3.4) implies $\left\langle L_{s}, i L_{t}\right\rangle=0$, which shows that $L: M^{2} \rightarrow \mathbf{C}_{1}^{2}$ is Lagrangian. This proves statement (i).

Conversely, it follows from (3.4) and Lemma 2.3 that

$$
\left\langle L_{s}, i L_{t}\right\rangle=\left\langle f^{\prime}, f-p\right\rangle\left\langle z, i z^{\prime}\right\rangle \text {. }
$$

Therefore, if $L_{f p z}$ is Lagrangian, then we find

$$
\left\langle f^{\prime}(s), f(s)-p(t)\right\rangle\left\langle z(t), i z^{\prime}(t)\right\rangle=0
$$

identically. Let us assume that $\left\langle z(t), i z^{\prime}(t)\right\rangle \neq 0$ on an open subinterval $\tilde{I} \subset I_{1}$, then we have $\left\langle f^{\prime}(s), f(s)-p(t)\right\rangle=0$ holds for all $s$ on $\tilde{I}$. Thus we get

$$
|f(s)-p(t)|_{s}^{2}=0
$$


which implies that, for each $t \in I_{2}$, the curve $f$ is contained in a circle centered at $p(t)$ in $\mathbf{C}^{*}$. This is impossible unless $p$ is a constant. Hence, when $f=f(s)$ contains no circular arcs, we get $\left\langle z, i z^{\prime}\right\rangle=0$, which is nothing but $z=z(t)$ is a Legendre curve in $S_{2}^{3}(1) \subset \mathbf{C}_{1}^{2}$.

Similarly, we also have the following.

Proposition 3.2. Let $f: I_{1} \rightarrow \mathbf{C}^{*}$ be a regular curve defined on an open interval $I_{1}$ and let $z: I_{2} \rightarrow H_{1}^{3}(-1) \subset \mathbf{C}_{1}^{2}$ be a unit speed space-like curve defined on an open interval $I_{2}$ containing 0 . Then, for any functions $p: I_{2} \rightarrow \mathbf{C}$, we have:

(i) If $z: I_{2} \rightarrow H_{1}^{3}(-1) \subset \mathbf{C}_{1}^{2}$ is a Legendre curve, then

$$
P_{f p z}(s, t)=f(s) z(t)-\int_{0}^{t} p(t) z^{\prime}(t) d t
$$

defines a Lagrangian isometric immersion of $M^{2}=\left(U_{f z}, g_{f p z}\right)$ into $\mathbf{C}_{1}^{2}$, where

$$
U_{f z}=\left\{(s, t) \in I_{1} \times I_{2}: f(s) \neq p(t)\right\}
$$

and $g_{f p z}$ is the Lorentzian metric given by

$$
g_{f p z}=-\left|f^{\prime}(s)\right|^{2} d s^{2}+|f(s)-p(t)|^{2} d t^{2} .
$$

(ii) Conversely, if $f$ contains no circular arcs and if $P_{f p z}$ defines a Lagrangian immersion, then $z: I_{2} \rightarrow H_{1}^{3}(-1) \subset \mathbf{C}_{1}^{2}$ is a Legendre curve in $H_{1}^{3}(-1)$.

Proof. This can be done in the same as Proposition 3.1.

\section{EXTRINSIC PROPERTIES.}

For a unit speed planar curve $f: I \rightarrow \mathbf{C}$ defined on an open interval $I$, we define the curvature function of $f$ on $I$ by

$$
\kappa(s)=\left\langle f^{\prime \prime}(s), i f^{\prime}(s)\right\rangle .
$$

We may express $f$ in polar form as $f=\rho(s) e^{i \theta(s)}$ for some real-valued function $\theta$.

Theorem 4.1. Let $f: I_{1} \rightarrow \mathbf{C}^{*}$ be a unit speed curve, $z: I_{2} \rightarrow S_{2}^{3}(1) \subset \mathbf{C}_{1}^{2} a$ unit speed time-like Legendre curve, $p(t)$ a complex-valued function defined on $I_{2}$ containing 0 , and let $L=L_{f p z}:\left(U_{f z}, g_{f p z}\right) \rightarrow \mathbf{C}_{1}^{2}$ be the Lagrangian isometric immersion defined by

$$
L_{f p z}(s, t)=f(s) z(t)-\int_{0}^{t} p(t) z^{\prime}(t) d t .
$$

Then we have:

(1) $L_{s}=\partial L / \partial s$ is an eigenvector of the shape operator $A_{J L_{s}}$ with eigenvalue $\kappa$, where $\kappa$ is the curvature function of the plane curve $f=f(s)$.

(2) $L_{t}=\partial L / \partial t$ is an eigenvector of the shape operator $A_{J L_{t}}$ if and only if $p$ is constant and $f(s)$ is a part of a line through the point $p$, i.e., $f(s)=c s+p$ for some $c \in \mathbf{C}$ with $|c|=1$.

(3) $L$ is a totally geodesic immersion if and only if

(3.1) $p$ is constant, $f=f(s)$ is a part of a line through $p$, and

(3.2) $z: I_{2} \rightarrow S_{2}^{3}(1) \subset \mathbf{C}_{1}^{2}$ is a geodesic in $S_{2}^{3}(1)$. 
Proof. Under the hypothesis, it follows from Eq. (3.3) of Proposition 3.1 that the induced metric on $U_{f z}$ via $L_{f p z}$ is the following twisted product Lorentzian metric

$$
g_{f p z}=d s^{2}-|f(s)-p(t)|^{2} d t^{2} .
$$

From (4.2) we find

$$
\begin{aligned}
& L_{s}=\frac{\partial L}{\partial s}=f^{\prime}(s) z(t), \\
& L_{t}=\frac{\partial L}{\partial t}=(f(s)-p(t)) z^{\prime}(t), \\
& L_{s s}=f^{\prime \prime}(s) z(t), L_{s t}=f^{\prime}(s) z^{\prime}(t), \\
& L_{t t}=(f(s)-p(t)) z^{\prime \prime}(t)-p^{\prime}(t) z^{\prime}(t) .
\end{aligned}
$$

By applying (2.8), (3.3), (4.4) and Lemma 2.3, we find

$$
\begin{aligned}
& \left\langle L_{s s}, i L_{s}\right\rangle=\left\langle f^{\prime \prime}, i f^{\prime}\right\rangle=\kappa, \\
& \left\langle L_{s s}, i L_{t}\right\rangle=\left\langle L_{s t}, i L_{s}\right\rangle=0, \\
& \left\langle L_{t t}, i L_{s}\right\rangle=\left\langle L_{s t}, i L_{t}\right\rangle=\left\langle f^{\prime}, i p-i f\right\rangle, \\
& \left\langle L_{t t}, i L_{t}\right\rangle=|f-p|^{2}\left\langle z^{\prime \prime}, i z^{\prime}\right\rangle+\left\langle p^{\prime}, i f-i p\right\rangle .
\end{aligned}
$$

Since $f(s)$ is assumed to be of unit speed, we have $\left|f^{\prime}(s)\right|=1$. Let us put

$$
e_{1}=L_{s}, \quad e_{2}=\frac{L_{t}}{|f-p|} .
$$

Then $e_{1}, e_{2}$ form a pseudo-orthonormal frame according to (4.3). Hence, by using (2.2), (2.3), (4.3), (4.5) and Lemma 2.1, we find

$$
\begin{aligned}
& A_{J e_{1}}=\left(\begin{array}{cc}
\kappa(s) & 0 \\
0 & \frac{\left\langle f^{\prime}, i f-i p\right\rangle}{|f-p|^{2}}
\end{array}\right), \\
& A_{J e_{2}}=\left(\begin{array}{cc}
0 & \frac{\left\langle f^{\prime}, i p-i f\right\rangle}{|f-p|^{2}} \\
\frac{\left\langle f^{\prime}, i f-i p\right\rangle}{|f-p|^{2}} & \frac{\lambda(t)}{|f-p|}+\frac{\left\langle p^{\prime}, i p-i f\right\rangle}{|f-p|^{3}}
\end{array}\right),
\end{aligned}
$$

where $\lambda$ is the curvature function of $z$ in $S_{2}^{3}(1)$.

Clearly, statement (1) of this theorem follows from (4.7). For statement (2), we observe that the third equation in (4.5) implies that $L_{t}$ is an eigenvector of $A_{J L_{t}}$ if and only if

$$
\left\langle f^{\prime}(s), i p(s)-i f(t)\right\rangle=0
$$

holds identically. Since the later condition holds true if and only if the position vector of $\gamma_{p(t)}(s)=f(s)-p(t)$ is always tangent to the curve $\gamma_{p(t)}$ for any fixed $t$. Therefore, for each $t$ the curve $\gamma_{p(t)}$ is a part of a line through the origin of $\mathbf{C}$. Consequently, there exists a unit vector field $c(t)$ in $\mathbf{C}$ such that $f(s)-p(t)=c(t) s$. This implies that both $c$ and $p$ are constant. Thus we must have $f(s)=c s+p$, Consequently, $f(s)$ is a part of a line through $p$. This gives statement (2) of the theorem.

For statement (3), let us assume that $L: M^{2} \rightarrow \mathbf{C}_{1}^{2}$ is totally geodesic. Then statement (2) implies that $p$ is constant and $f=c s+p$ for some unit complex 
number $c$. Moreover, it follows from (4.8) that

$$
0=\lambda(t)|f(s)-p|^{2}+\left\langle p^{\prime}, i p-i f(s)\right\rangle=\lambda(t)|f(s)-p|^{2} .
$$

By applying these facts we conclude that $\lambda=0$, which shows that the Legendre curve $z$ has zero curvature in $S_{2}^{3}(1)$. Consequently, $z$ is a geodesic in $S_{2}^{3}(1)$.

The converse follows easily from (4.5) and Lemma 2.1. Thus we have statement (3) of the theorem.

Similarly, we have the following.

Theorem 4.2. Let $f: I_{1} \rightarrow \mathbf{C}^{*}$ be a unit speed curve, $z: I_{2} \rightarrow H_{1}^{3}(-1) \subset \mathbf{C}_{1}^{2} a$ unit speed space-like Legendre curve, $p$ a complex-valued function defined on $I_{2} \ni 0$, and $P=P_{f p z}:\left(U_{f z}, g_{f p z}\right) \rightarrow \mathbf{C}_{1}^{2}$ the Lagrangian isometric immersion defined by

$$
P_{f p z}(s, t)=f(s) z(t)-\int_{0}^{t} p(t) z^{\prime}(t) d t .
$$

Then we have:

(a) $P s=\partial P / \partial s$ is an eigenvector of the shape operator $A_{J P_{s}}$ with eigenvalue $\kappa$, where $\kappa$ is the curvature function of the plane curve $f=f(s)$.

(b) $P_{t}=\partial P / \partial t$ is an eigenvector of the shape operator $A_{J P_{t}}$ if and only if $p$ is constant and $f(s)$ is a part of a line through the point $p$.

(c) $P$ is a totally geodesic immersion if and only if

(c.1) $p$ is constant, $f=f(s)$ is a part of a line through $p$, and

(c.2) $z: I_{2} \rightarrow H_{1}^{3}(-1) \subset \mathbf{C}_{1}^{2}$ is a geodesic in $H_{1}^{3}(-1)$.

Proof. Under the hypothesis, it follows from (3.3) of Proposition 3.1 that the induced metric on $U_{f z}$ via $L_{f p z}$ is given by (3.8). By applying (2.8), (3.8), (4.10), Lemma 2.2 and Lemma 2.3, we find

$$
\begin{aligned}
& \left\langle P_{s s}, i P_{s}\right\rangle=-\left\langle f^{\prime \prime}, i f^{\prime}\right\rangle=-\kappa, \\
& \left\langle P_{s s}, i P_{t}\right\rangle=\left\langle P_{s t}, i P_{s}\right\rangle=0, \\
& \left\langle P_{t t}, i P_{s}\right\rangle=\left\langle P_{s t}, i P_{t}\right\rangle=\left\langle f^{\prime}, i f-i p\right\rangle, \\
& \left\langle P_{t t}, i P_{t}\right\rangle=\lambda|f-p|^{2}+\left\langle p^{\prime}, i p-i f\right\rangle .
\end{aligned}
$$

As in the proof of Theorem 4.1, if we put

$$
e_{1}=L_{s}, \quad e_{2}=\frac{L_{t}}{|f-p|} .
$$

Then $e_{1}, e_{2}$ form a pseudo-orthonormal frame. Hence, after using $(2.2),(2.3),(3.8)$, (4.11), (4.12) and Lemma 2.1, we obtain

$$
\begin{gathered}
A_{J e_{1}}=\left(\begin{array}{cc}
\kappa(s) & 0 \\
0 & \frac{\left\langle f^{\prime}, i f-i p\right\rangle}{|f-p|^{2}}
\end{array}\right), \\
A_{J e_{2}}=\left(\begin{array}{cc}
0 & \frac{\left\langle f^{\prime}, i p-i f\right\rangle}{|f-p|^{2}} \\
\frac{\left\langle f^{\prime}, i f-i p\right\rangle}{|f-p|^{2}} & \frac{\lambda(t)}{|f-p|}+\frac{\left\langle p^{\prime}, i p-i f\right\rangle}{|f-p|^{3}}
\end{array}\right),
\end{gathered}
$$

where $\lambda$ is the curvature function of $z$ in $S_{2}^{3}(1)$. Now, we may conclude the theorem in the same way as Theorem 4.1. 


\section{Classification of Lagrangian surfaces of Constant Curvature in $\mathbf{C}_{1}^{2}$}

In this section we completely classify Lagrangian surfaces of constant curvature which are constructed via our construction method.

Theorem 5.1. Let $f: I_{1} \rightarrow \mathbf{C}^{*}$ be a unit speed curve in $\mathbf{C}^{*}, p: I_{2} \rightarrow \mathbf{C}$ a unit speed curve in $\mathbf{C}$ defined on an open interval $I_{2} \ni 0$ such that $p$ does not meet the curve $f$, and let $z: I_{2} \rightarrow S_{2}^{3}(1) \subset \mathbf{C}_{1}^{2}$ be a unit speed time-like Legendre curve. Then the Lagrangian surface defined by

$$
L=L_{f p z}(s, t)=f(s) z(t)-\int_{0}^{t} p(t) z^{\prime}(t) d t
$$

is of constant Gauss curvature $K$ if and only if, up to reparametrization of $s$ and dilations and rigid motions of of $\mathbf{C}_{1}^{2}$, one of the following eight cases occurs:

(a) $K=0$ and

$$
L=r e^{i s / r} z(t)
$$

where $r$ is a positive number.

(b) $K=0$ and

$$
L=s z(t)-\int_{0}^{t} p(t) z^{\prime}(t) d t
$$

where $p$ is a real-valued function.

(c) $K=0$ and

$$
L=\frac{r s^{1+i / r}}{\sqrt{1+r^{2}}} z(t)
$$

where $r$ is a nonzero real number.

(d) $K=1$ and

$$
L=\frac{1}{2}\left\{z(t) e^{2 i s}+\int_{0}^{t} e^{-2 i B(t)} z^{\prime}(t) d t\right\}
$$

where $B(t)$ is a real-valued function;

(e) $K=1$ and

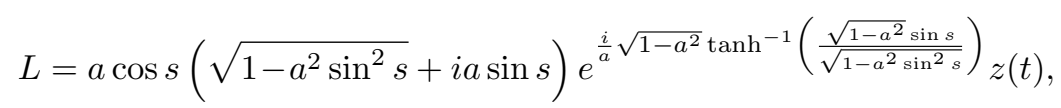

where $a$ is a nonzero real number.

(f) $K=-1$ and

$$
L=\left(a^{2} e^{2 s}-i a e^{s} \sqrt{1-a^{2} e^{2 s}}\right) e^{\frac{i}{a} \sqrt{e^{-2 s}-a^{2}}} z(t),
$$

where $a$ is a positive real number.

(g) $K=-1$, and

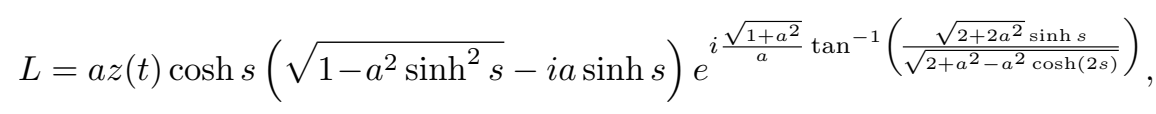

where $a$ is a real number satisfying $0<a<1$;

(h) $K=-1$, and

$$
L=a z(t) \sinh s e^{ \pm \frac{i}{a} \int_{0}^{s} \sqrt{\operatorname{csch}^{2} s-a^{2} \operatorname{coth}^{2} s} d s,}
$$

where $a$ is a positive number. 
Proof. Suppose that $f: I_{1} \rightarrow \mathbf{C}^{*}$ is a unit speed curve, $p(t)$ is a complex-valued function defined on an open interval $I_{2} \ni 0$ such that $p$ does not meet the curve $f$, and $z: I_{2} \rightarrow S^{3}(1) \subset \mathbf{C}_{1}^{2}$ is a unit speed time-light Legendre curve. Then the induced metric $g_{f p z}$ via $L_{f p z}$ is given by

$$
g_{f p z}=d s^{2}-|f(s)-p(t)|^{2} d t^{2} .
$$

Thus the Gauss curvature $K$ of $\left(U_{f z}, g_{f p z}\right)$ satisfies

$$
G_{s s}=-K G, \quad G=|f(s)-p(t)| .
$$

Case (1): $K=0$. Solving (5.2) gives

$$
|f(s)-p(t)|^{2}=(A(t) s+B(t))^{2}
$$

for some real-valued functions $A(t)$ and $B(t)$.

CAse (1.1): $A(t)=0$. In this case, (5.3) reduces to $|f(s)-p(t)|^{2}=B^{2}(t)$. Thus we find

$$
\left\langle f^{\prime}(s), f(s)-p(t)\right\rangle=0
$$

which implies that

$$
\left\langle f^{\prime}(s), p^{\prime}(t)\right\rangle=\left\langle f^{\prime \prime}(s), p^{\prime}(t)\right\rangle=0 .
$$

If $f^{\prime \prime}=0$ holds identically, we get $f(s)=a s+b$ for some complex numbers $a, b$ with $|a|=1$. Substituting this into (5.4) gives

$$
\langle a, p(t)\rangle=\langle a, a s+b\rangle=s+\langle a, b\rangle
$$

which is impossible. Therefore we must have $f^{\prime \prime}(s) \neq 0$.

Since $\left|f^{\prime}(s)\right|=1$, we have $\left\langle f^{\prime}, f^{\prime \prime}\right\rangle=0$. Thus $f^{\prime \prime} \neq 0$ and (5.5) imply that $p$ is constant. Hence, by (5.3), $B$ is a positive real number, say $r$. Consequently, after applying a suitable translation on $s$, we obtain $f(s)=p+r e^{i s / r}, p \in \mathbf{C}$. After substituting these into (5.1) we obtain case (a) of the theorem.

CASE (1.2): $A(t) \neq 0$. We obtain from (5.3) and $\left|f^{\prime}\right|=1$ that

$$
\begin{aligned}
& \left\langle f^{\prime}(s), f(s)-p(t)\right\rangle=A^{2}(t) s+A(t) B(t), \\
& \left\langle f^{\prime \prime}(s), f(s)-p(t)\right\rangle=A^{2}(t)-1 .
\end{aligned}
$$

CAse (1.2.a): $f^{\prime \prime}=0$. We have $f(s)=a s+c_{1}$ for some complex numbers $a, c_{1}$ with $a=e^{i \psi}, \psi \in \mathbf{R}$. Thus, after applying some suitable rotation and translation on $\mathbf{C}$, we get $f(s)=s$. Also, it follows from (5.7) that $A^{2}=1$. Without loss of generality, we may assume that $A=1$. Now, by substituting these into (5.3) we conclude that $p(t)$ is the real-valued function satisfying $p(t)=-B(t)$. Consequently, after applying these facts and (5.1) we get case (b).

CASE (1.2.b): $f^{\prime \prime} \neq 0$. Since $f^{\prime \prime}(s)=i \kappa f^{\prime}$ with $\kappa \neq 0$, we obtain from (5.6) and (5.7) that

$$
f(s)=p(t)+\left(A^{2}(t) s+A(t) B(t)\right) f^{\prime}(s)+\left(\frac{A^{2}(t)-1}{\kappa(s)}\right) i f^{\prime}(s) .
$$

By differentiating (5.8) with respect to $s$ we find

$$
\left(\frac{1}{\kappa^{2}}\right)^{\prime}=\frac{2 A^{2}(t) s}{1-A^{2}(t)}+\frac{2 A(t) B(t)}{1-A^{2}(t)}
$$


which implies

$$
\left(\frac{1}{\kappa^{2}(s)}\right)^{\prime \prime}=\frac{2 A^{2}(t)}{1-A^{2}(t)} .
$$

Therefore there exists a nonzero real number $a$ such that

$$
\left(\frac{1}{\kappa^{2}}\right)^{\prime \prime}=2 a, \quad A^{2}(t)=a\left(1-A^{2}(t)\right) .
$$

Thus we have

$$
\frac{1}{\kappa^{2}}=a s^{2}+c_{1} s+c_{2}
$$

for some real numbers $c_{1}, c_{2}$. Hence, after applying a suitable translation on $s$, we obtain

$$
\frac{1}{\kappa^{2}}=a s^{2}+b, \quad A^{2}=\frac{a}{a+1}
$$

for some real number $b$.

From (5.9) and (5.11), we get $B=0$. Hence (5.8) reduces to

$$
f(s)=p+\left(\frac{a s}{a+1}-\frac{i}{(a+1) \kappa(s)}\right) f^{\prime}(s),
$$

which shows that $p$ is constant.

If $b=0$, we get $a>0$. If we put $a=r^{2}, r>0$, then we find from (5.11) that $\kappa=1 /(r s)$. Substituting this into (5.12) gives

$$
f(s)=p+\frac{r(r-i) s f^{\prime}(s)}{\left(r^{2}+1\right)} .
$$

After solving this differential equation we obtain $f(s)=p+\mu s^{1+i / r}$ for some complex numbers $\mu$. Since $\left|f^{\prime}\right|=1$, we have $|\mu|=r / \sqrt{1+r^{2}}$. Therefore, after applying a suitable rigid motion on $\mathbf{C}$, we obtain

$$
f(s)=p+\frac{r s^{1+i / r}}{\sqrt{1+r^{2}}} .
$$

After substituting this into (5.1) we obtain case (c).

If $b \neq 0$, then we obtain from (5.11) and (5.12) that

$$
f^{\prime}(s)=\frac{(a+1)\left(a s \pm i \sqrt{a s^{2}+b}\right)}{a(a+1) s^{2}+b}(f(s)-p)
$$

which implies

$$
|f-p|^{2}=\frac{a(1+a) s^{2}+b}{(1+a)^{2}} .
$$

By comparing (5.3) and (5.14), we obtain $b=0$ which is a contradiction.

CASE (2): $K=1$. Solving (5.2) gives

$$
|f(s)-p(t)|=A(t) \cos (s+B(t))
$$

for some real-valued functions $A(t) \neq 0$ and $B(t)$. Thus we find

$$
\begin{aligned}
& \left\langle f^{\prime}(s), f(s)-p(t)\right\rangle=-\frac{1}{2} A^{2} \sin (2 s+2 B), \\
& \left\langle f^{\prime \prime}(s), f(s)-p(t)\right\rangle=-A^{2} \cos (2 s+2 B)-1 .
\end{aligned}
$$


If $f^{\prime \prime}=0$, then (5.17) yields

$$
A^{2}(t) \cos (2 s+2 B(t))+1=0
$$

which is impossible. Thus $f^{\prime \prime} \neq 0$, and $f^{\prime}(s), f^{\prime \prime}(s) / \kappa$ form an orthonormal frame. Hence, after applying (5.16) and (5.17), we find

$$
f(s)=p(t)-\left\{\frac{A^{2}}{2} \sin (2 s+2 B)+\frac{i}{\kappa}\left(A^{2} \cos (2 s+2 B)+1\right)\right\} f^{\prime}(s) .
$$

Now, by differentiating (5.18) with respect to $s$ we get

$$
\kappa^{\prime}(s)\left(1+A^{2}(t) \cos (2 s+2 B(t))\right)=\frac{1}{2}\left(\kappa^{3}-4 \kappa\right) A^{2}(t) \sin (2 s+2 B(t)),
$$

CASE (2.a): $\kappa$ is constant. In this case, (5.19) implies $\kappa^{2}=4$. Hence, $f$ lies in a circle of radius $1 / 2$. Without loss of generality, we may assume $\kappa=2$. Since $f(s)$ is a unit speed curve, we may also put

$$
f(s)=q+\frac{e^{2 i s}}{2}
$$

for some $q \in \mathbf{C}$. Substituting this into (5.16) and (5.17) gives

$$
\begin{aligned}
\left\langle i e^{2 i s}, q-p(t)\right\rangle & =-\frac{1}{2} A^{2} \sin (2 s+2 B), \\
2\left\langle e^{2 i s}, q-p(t)\right\rangle & =A^{2} \cos (2 s+2 B) .
\end{aligned}
$$

These two equations imply that

$$
2 q=2 p(t)+A^{2}(t) e^{-2 i B} .
$$

Combining this with (5.20) yields

$$
f(s)=p(t)+\frac{1}{2}\left(A^{2}(t) e^{-2 i B}+e^{2 i s}\right)
$$

Thus

$$
4|f(s)-p|^{2}=\left(A^{2}-1\right)^{2}+4 A^{2} \cos ^{2}(s+B) .
$$

Now, by comparing (5.23) and (5.15) we find $A=1$. Hence (5.22) reduces to

$$
f(s)=p(t)+\frac{e^{-2 i B}+e^{2 i s}}{2} .
$$

If $B=b$ is constant, then (5.24) implies that $p$ is constant. Thus, after applying (5.1) and (5.24), we obtain a special case of case (d) of the theorem.

If $B$ is non-constant, then we find from (5.20) and (5.21) that

$$
f(s)=q+\frac{e^{2 i s}}{2}, \quad p(t)=q-\frac{e^{-2 i B(t)}}{2},
$$

where $q \in \mathbf{C}$ and $B(t)$ is an arbitrary real-valued function. Consequently, after applying (5.1) and (5.25), we obtain case (d) for non-constant $B$.

CASE (2.b): $\kappa$ is non-constant. After solving (5.19) we find

$$
\kappa(s)= \pm \frac{2\left(1+A^{2} \cos (2 s+2 B)\right)}{\sqrt{\left(1+A^{2} \cos (2 c s+2 B)\right)^{2}-e^{-8 b}}}
$$


for some real number $b$. Without loss of generality, we may assume that

$$
\kappa(s)=\frac{2\left(1+A^{2} \cos (2 s+2 B)\right)}{\sqrt{\left(1+A^{2} \cos (2 s+2 B)\right)^{2}-e^{-8 b}}} .
$$

Since $\kappa$ is non-constant, we see from (5.26) that $A \neq 0$ and $B$ is constant. Thus, after applying a suitable translation on $s$, we get $B=0$. Thus, by substituting (5.26) into (5.18) we find

$$
f=p-\frac{f^{\prime}}{2}\left\{A^{2} \sin (2 s)+i \sqrt{\left(1+A^{2} \cos (2 s)\right)^{2}-e^{-8 b}}\right\} .
$$

Thus, by applying $\left|f^{\prime}(s)\right|=1$, we find from (5.27) that

$$
|f-p|^{2}=A^{2} \cos ^{2} s+\frac{\left(A^{2}-1\right)^{2}-e^{-8 b}}{4} .
$$

Comparing (5.15) and (5.28) gives $\left(A^{2}-1\right)^{2}=e^{-8 b}$. Thus $A$ is a real number say $a$, which gives $e^{-8 b}=\left(a^{2}-1\right)^{2}$. Without loss of generality we may assume $a>0$. Therefore (5.26) and (5.27) yield

$$
\begin{aligned}
& f=p-\frac{a}{2} f^{\prime}\left\{a \sin (2 s)+2 i \cos s \sqrt{1-a^{2} \sin ^{2} s}\right\}, \\
& \kappa(s)=\frac{\left(1+a^{2} \cos (2 s)\right) \sec s}{a \sqrt{1-a^{2} \sin ^{2} s}} .
\end{aligned}
$$

From (5.29) we obtain

$$
f^{\prime}(s)=\frac{(p-f(s))}{a}\left(a \tan s-i \sqrt{\sec ^{2} s-a^{2} \tan ^{2} s}\right) .
$$

After solving (5.31) we obtain

$$
f-p=\alpha \cos s\left(\sqrt{1-a^{2} \sin ^{2} s}+i a \sin s\right) e^{\frac{i}{a} \sqrt{1-a^{2}} \tanh ^{-1}\left(\frac{\sqrt{1-a^{2}} \sin s}{\sqrt{1-a^{2} \sin ^{2} s}}\right)}
$$

for some $\alpha \in \mathbf{R}$. It follows from (5.1) and (5.32) that $L$ is given by

$$
L=\alpha \cos s\left(\sqrt{1-a^{2} \sin ^{2} s}+i a \sin s\right) e^{\frac{i}{a} \sqrt{1-a^{2}} \tanh ^{-1}\left(\frac{\sqrt{1-a^{2}} \sin s}{\sqrt{1-a^{2} \sin ^{2} s}}\right)} z(t) .
$$

Now, by applying (5.33) and $\langle z, z\rangle=\left|f^{\prime}(s)\right|=1$, we get $\left\langle L_{s}, L_{s}\right\rangle=\alpha^{2} / a^{2}$. Thus we may find from (3.3) of Proposition 3.1 that $q=a$. Consequently, we obtain case (e) of the theorem.

Case (3): $K=-1$. Solving (5.2) gives

$$
|f(s)-p(t)|=\alpha(t) \cosh s+\beta(t) \sinh s
$$

for some real-valued functions $\alpha(t)$ and $\beta(t)$.

We divide this into three cases.

CAse (3.1): $\alpha^{2}=\beta^{2}$. In this case, (5.34) becomes

$$
|f(s)-p(t)|=a(t) e^{ \pm s},
$$

where $a$ is a positive function. Without loss of generality, we may assume that

$$
|f(s)-p(t)|=a(t) e^{s}
$$


for some nonzero real-valued function. Thus we find

$$
\begin{aligned}
& \left\langle f^{\prime}(s), f(s)-p(t)\right\rangle=a^{2} e^{2 s} \\
& \left\langle f^{\prime \prime}(s), f(s)-p(t)\right\rangle=2 a^{2} e^{2 s}-1 .
\end{aligned}
$$

If $f^{\prime \prime}=0$, then (5.37) gives $2 a^{2}(t) e^{2 s}=1$ which is impossible. Hence we must have $f^{\prime \prime}=i \kappa f^{\prime} \neq 0$. Thus, by applying (5.36) and (5.37), we find

$$
f(s)=p(t)+\left\{a^{2} e^{2 s}+\frac{i}{\kappa}\left(2 a^{2} e^{2 s}-1\right)\right\} f^{\prime}(s)
$$

which shows that $p$ is a complex number. After differentiating (5.38) with respect to $s$ and applying $f^{\prime \prime}=i \kappa f^{\prime}$, we find

$$
\kappa^{\prime}(s)=\frac{a^{2}(t) e^{2 s} \kappa(s)\left(4+\kappa^{2}(s)\right)}{2 a^{2}(t) e^{2 s}-1} .
$$

After differentiating (5.39) with respect to $t$, we find $a^{\prime}(t)=0$. Hence $a(t)$ is a positive real number. Now, by solving (5.39) for $\kappa$ we obtain

$$
\kappa= \pm \frac{2 b^{2}\left(2 a^{2} e^{2 s}-1\right)}{\sqrt{1-b^{4}\left(1-2 a^{2} e^{2 s}\right)^{2}}}
$$

for some positive real number $b$. Without loss of generality, we may assume that

$$
\kappa=\frac{2 b^{2}\left(2 a^{2} e^{2 s}-1\right)}{\sqrt{1-b^{4}\left(1-2 a^{2} e^{2 s}\right)^{2}}} .
$$

After substituting this into (5.38) we obtain

$$
f(s)=p+\left\{a^{2} e^{2 s}+i \frac{\sqrt{1-b^{4}\left(1-2 a^{2} e^{2 s}\right)^{2}}}{2 b^{2}}\right\} f^{\prime}(s),
$$

It follows from (5.40) and $\left|f^{\prime}(s)\right|=1$ that

$$
|f(s)-p(t)|^{2}=a^{4} e^{4 s}+\frac{1-\left(1-2 a^{2} e^{2 s}\right)^{2}}{2 b^{2}}
$$

By comparing (5.35) and (5.41), we find $b=1$. Therefore (5.40) becomes

$$
f(s)=p+\frac{1}{2}\left\{2 a^{2} e^{2 s}+i \sqrt{1-\left(1-2 a^{2} e^{2 s}\right)^{2}}\right\} f^{\prime}(s)
$$

Now, after we solve $(5.42)$ for $f(s)$, we obtain

$$
f(s)=p+\frac{\delta}{a} e^{s}\left(a e^{s}-i \sqrt{1-a^{2} e^{2 s}}\right) e^{\frac{i}{a} \sqrt{e^{-2 s}-a^{2}}} z(t),
$$

for some constant $\delta$. It follows from $\left|f^{\prime}(s)\right|=1$ and (5.43) that $\delta=a^{2}$. Therefore, we conclude from (5.1) and (5.43) that

$$
L=\left(a^{2} e^{2 s}-i a e^{s} \sqrt{1-a^{2} e^{2 s}}\right) e^{\frac{i}{a} \sqrt{e^{-2 s}-a^{2}}} z(t) .
$$

This gives case (f) of the theorem.

CASE (3.2): $\alpha^{2}>\beta^{2}$. If we put

$$
A(t)=\sqrt{\alpha^{2}-\beta^{2}}, \quad \alpha=A \cosh \eta, \quad \beta=A \sinh \eta
$$

for some real-valued function $\eta(t)$, then (5.34) becomes

$$
|f(s)-p(t)|=A(t) \cosh (s+\eta),
$$


which implies that

$$
\begin{aligned}
& \left\langle f^{\prime}(s), f(s)-p(t)\right\rangle=\frac{A^{2}(t)}{2} \sinh (2 s+2 \eta), \\
& \left\langle f^{\prime \prime}(s), f(s)-p(t)\right\rangle=A^{2}(t) \cosh (2 s+2 \eta)-1 .
\end{aligned}
$$

From (5.46) we get $f^{\prime \prime}=i \kappa f^{\prime} \neq 0$. So, we (5.45) and (5.46) imply that

$$
f(s)=p(t)+\left\{\frac{1}{2} A^{2}(t) \sinh (2 s+2 \eta)+\frac{i}{\kappa}\left(A^{2}(t) \cosh (2 s+2 \eta)-1\right)\right\} f^{\prime}(s) .
$$

Differentiating this equation with respect to $s$ gives

$$
2 \kappa^{\prime}(s)\left(1-A^{2}(t) \cosh (2 s+2 \eta)\right)+\kappa(s) A^{2}(t)\left(4+\kappa^{2}\right) \sinh (2 s+2 \eta)=0,
$$

which can be written as

$$
A^{2}(t)=\frac{2 \kappa^{\prime}(s)}{2 \kappa^{\prime} \cosh (2 s+2 \eta)-\kappa\left(4+\kappa^{2}\right) \sinh (2 s+2 \eta)} .
$$

By differentiating (5.49) with respect $s$ gives

$$
\operatorname{coth}(2 s+2 \eta)=\frac{\left(4+\kappa^{2}\right) \kappa^{\prime \prime}-3 \kappa \kappa^{\prime 2}}{2\left(4+\kappa^{2}\right) \kappa^{\prime}(s)} .
$$

Since the right-hand-side of (5.49) depends only on $s, \eta(t)$ is a real number. Thus, after applying a suitable translation in $s$, we have $\eta=0$. Also, we see follows from (5.49) that $A$ is a positive real number. Consequently, (5.48) yields

$$
2 \kappa^{\prime}(s)\left(1-a^{2} \cosh (2 s)\right)+\kappa(s) a^{2}\left(4+\kappa^{2}\right) \sinh (2 s)=0,
$$

After solving (5.51) we get

$$
\kappa(s)= \pm \frac{2 b^{2}\left(1-a^{2} \cosh (2 s)\right)}{\sqrt{1-b^{4}\left(1-a^{2} \cosh (2 s)\right)^{2}}},
$$

for some positive number $b$. Without loss of generality, we may assume that

$$
\kappa(s)=\frac{2 b^{2}\left(1-a^{2} \cosh (2 s)\right)}{\sqrt{1-b^{4}\left(1-a^{2} \cosh (2 s)\right)^{2}}} .
$$

By substituting this into (5.47) and applying $A=a$ and $\eta=0$, we get

$$
f(s)=p+\left\{\frac{a^{2}}{2} \sinh (2 s)-\frac{i \sqrt{1-b^{4}\left(1-a^{2} \cosh (2 s)\right)^{2}}}{2 b^{2}}\right\} f^{\prime}(s) .
$$

Since $\left|f^{\prime}\right|=1$, it follows from (5.53) that

$$
|f(s)-p|^{2}=\frac{1}{4}\left(\frac{1}{b^{4}}+2 a^{2} \cosh (2 s)-a^{4}-1\right) .
$$

On the other hand, we also have

$$
|f-p|^{2}=a^{2} \cosh ^{2}(s) .
$$

Now, by comparing this with (5.54), we obtain $b^{2}=1 /\left(1+a^{2}\right)$. Therefore, we obtain from (5.53) that

$$
f(s)-p=\frac{1}{2}\left\{a^{2} \sinh (2 s)-i \sqrt{\left(1+a^{2}\right)^{2}-\left(1-a^{2} \cosh (2 s)\right)^{2}}\right\} f^{\prime}(s) .
$$


After (5.55) we obtain

$$
f(s)-p=\gamma \cosh s\left(\sqrt{1-a^{2} \sinh ^{2} s}-i a \sinh s\right) e^{i \frac{\sqrt{1+a^{2}}}{a} \tan ^{-1}\left(\frac{\sqrt{2+2 a^{2}} \sinh s}{\sqrt{2+a^{2}-a^{2} \cosh (2 s)}}\right)}
$$

for some nonzero real number $\gamma$.

From (5.56) we get $|f-p|^{2}=r^{2} \cosh ^{2} s$. Comparing this with $|f-p|^{2}=a^{2} \cosh ^{2} s$ from before gives $r=a$. Consequently, we find case (g) from (5.1) and (5.56).

CASE (3.3): $\alpha^{2}<\beta^{2}$. If we put

$$
A(t)=\sqrt{\beta^{2}-\alpha^{2}}, \quad \alpha=A \sinh \theta, \quad \beta=A \cosh \theta,
$$

then (5.34) becomes

$$
|f(s)-p(t)|=A(t) \sinh (s+\theta(t))
$$

for some real-valued function $\theta(t)$. Thus we find

$$
\begin{aligned}
& \left\langle f^{\prime}(s), f(s)-p(t)\right\rangle=\frac{A^{2}(t)}{2} \sinh (2 s+2 \theta), \\
& \left\langle f^{\prime \prime}(s), f(s)-p(t)\right\rangle=A^{2}(t) \cosh (2 s+2 \theta)-1 .
\end{aligned}
$$

If $f^{\prime \prime}=0$, then $(5.59)$ gives

$$
A^{2}(t) \cosh (2 s+2 \theta)=1
$$

for all $t$ which is impossible. So, we must have $f^{\prime \prime}=i \kappa f^{\prime} \neq 0$. Hence it follows from (5.58) and (5.59) that

$$
f(s)=p(t)+\left\{\frac{1}{2} A^{2} \sinh (2 s+2 \theta)+\frac{i}{\kappa}\left(A^{2} \cosh (2 s+2 \theta)-1\right)\right\} f^{\prime}(s),
$$

which is exactly (5.47). Therefore, we can apply exactly the same way as in case (3.2) to conclude that $\theta=0, A(t)$ is a positive real number, say $a$, and $p(t)$ is a complex number. Hence in views of (5.57) we may put

$$
f(s)-p=a \sinh s e^{i \theta}
$$

for some real-valued function $\theta=\theta(s, t)$. From (5.61) and $\left|f^{\prime}(s)\right|=1$, we find

$$
a^{2} \theta_{s}^{2} \sinh ^{2} s=1-a^{2} \cosh ^{2} s,
$$

which implies that $a^{2} \leq 1$ due to $\cosh s \geq 1$.

If $a^{2}=1$, then $(5.62)$ becomes $\left(1+\bar{a}^{2} \theta_{s}^{2}\right) \sinh ^{2} s=0$, which is impossible. So, we must have $0<a<1$. Hence we find from (5.62) that

$$
\theta_{s}= \pm \frac{1}{a} \sqrt{\operatorname{csch}^{2} s-a^{2} \operatorname{coth}^{2} s}
$$

If we put

$$
\varphi(s)= \pm \frac{1}{a} \int_{0}^{s} \sqrt{\operatorname{csch}^{2} s-a^{2} \operatorname{coth}^{2} s} d s
$$

then we obtain from (5.63) that

$$
\theta(s, t)=\varphi(s)+\psi(t)
$$

for some real-valued function $\psi(t)$. Substituting this into (5.61) gives

$$
f(s)-p=a \sinh s e^{i\left( \pm \frac{1}{a} \int_{0}^{s} \sqrt{\operatorname{csch}^{2} s-a^{2} \operatorname{coth}^{2} s} d s+\psi(t)\right)}
$$


which shows that $\psi$ is a real number. Hence, after applying a suitable translation on $s$, we have $\psi=0$. Hence(5.65) reduces to

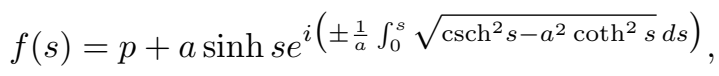

where $p$ is a complex number, $a$ is a nonzero real number and $r$ is a real number. Consequently, we obtain case (h) after combining (5.1) and (5.66).

The converse can be verified by straight-forward computation.

Similarly, we also have the following.

Theorem 5.2. Let $f: I_{1} \rightarrow \mathbf{C}^{*}$ be a unit speed curve, $z: I_{2} \rightarrow H_{1}^{3}(-1) \subset \mathbf{C}_{1}^{2}$ a unit speed space-like Legendre curve, and $p=p(t)$ a complex-valued function defined on $K$. Then the Lagrangian immersion $L_{f p z}:\left(U_{f z}, g_{f p z}\right) \rightarrow \mathbf{C}_{1}^{2}$ defined by

$$
P=P_{f p z}(s, t)=f(s) z(t)-\int_{0}^{t} p(t) z^{\prime}(t) d t
$$

is of constant Gauss curvature $K$ if and only if, up to reparametrization of $s$ and dilations and rigid motions of of $\mathbf{C}_{1}^{2}$, one of the following eight cases occurs:

(a) $K=0$ and

$$
P=r e^{i s / r} z(t)
$$

(b) $K=0$ and

$$
P=s z(t)-\int_{0}^{t} p(t) z^{\prime}(t) d t
$$

where $p$ is a real-valued function;

(c) $K=0$ and

where $r$ is a nonzero real number.

$$
P=\frac{r s^{1+i / r}}{\sqrt{1+r^{2}}} z(t)
$$

(d) $K=1$ and

$$
P=\frac{1}{2}\left\{z(t) e^{2 i s}+\int_{0}^{t} e^{-2 i B(t)} z^{\prime}(t) d t\right\}
$$

where $B(t)$ is a real-valued function;

(e) $K=1$ and

$$
P=a \cos s\left(\sqrt{1-a^{2} \sin ^{2} s}+i a \sin s\right) e^{\frac{i}{a} \sqrt{1-a^{2}} \tanh ^{-1}\left(\frac{\sqrt{1-a^{2}} \sin s}{\sqrt{1-a^{2} \sin ^{2} s}}\right)} z(t),
$$

where $a$ is a nonzero real number.

(f) $K=-1$ and

$$
P=\left(a^{2} e^{2 s}-i a e^{s} \sqrt{1-a^{2} e^{2 s}}\right) e^{\frac{i}{a} \sqrt{e^{-2 s}-a^{2}}} z(t),
$$

where $a$ is a positive real number.

(g) $K=-1$, and

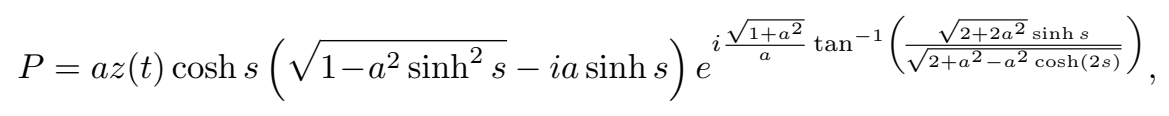

where $a$ is a real number satisfying $0<a<1$; 
(h) $K=-1$, and

$$
P=a z(t) \sinh s e^{ \pm \frac{i}{a} \int_{0}^{s} \sqrt{\operatorname{csch}^{2} s-a^{2} \operatorname{coth}^{2} s} d s},
$$

where $a$ is a positive number.

Proof. This can be proved exactly in the same was as Theorem 5.1.

\section{Classification of minimal Lagrangian surfaces in $\mathbf{C}_{1}^{2}$.}

Let $M$ be a Lagrangian surface in $\mathbf{C}_{1}^{2}$. If $e_{1}, e_{2}$ be a pseudo-orthonormal frame on $M$, then the mean curvature vector $H$ of $M$ in $\mathbf{C}_{1}^{2}$ is given by

$$
H=\frac{1}{2} \sum_{j=1}^{2} \epsilon_{j} h\left(e_{j}, e_{j}\right), \quad \epsilon_{j}=\left\langle e_{j}, e_{j}\right\rangle, j=1,2 .
$$

The Lagrangian surface $M$ is called minimal if $H=0$ holds identically.

The following are two examples of Lagrangian minimal surfaces in $\mathbf{C}_{1}^{2}$.

Example 6.1. Consider the surface $M_{1}$ in $\mathbf{C}_{1}^{2}$ defined by

$$
\left(a\left(\cosh \left(\frac{s}{a}\right)+i \sinh \left(\frac{s}{a}\right)\right) \sinh t, a\left(\cosh \left(\frac{s}{a}\right)+i \sinh \left(\frac{s}{a}\right)\right) \cosh t\right) .
$$

It is direct to verify that $M_{1}$ is a Lagrangian minimal surface in $\mathbf{C}_{1}^{2}$ whose induced metric is given by

$$
g_{1}=\left(\cosh ^{2} s\right)\left(-d s^{2}+d t^{2}\right) .
$$

Example 6.2. Consider the surface $M_{-1}$ in $\mathbf{C}_{1}^{2}$ defined by

$$
\left(a\left(\cosh \left(\frac{s}{a}\right)+i \sinh \left(\frac{s}{a}\right)\right) \cosh t, a\left(\cosh \left(\frac{s}{a}\right)+i \sinh \left(\frac{s}{a}\right)\right) \sinh t\right) .
$$

It is direct to verify that $M_{-1}$ is also a Lagrangian minimal surface in $\mathbf{C}_{1}^{2}$ whose induced metric is given by

$$
g_{1}=\left(\cosh ^{2} s\right)\left(d s^{2}-d t^{2}\right) .
$$

In this section we classify all minimal Lagrangian surfaces in $\mathbf{C}_{1}^{2}$ obtained by the construction method given in this paper.

Theorem 6.1. Let $f: I_{1} \rightarrow \mathbf{C}^{*}$ be a unit speed curve, $z: I_{2} \rightarrow S_{2}^{3}(1) \subset \mathbf{C}_{1}^{2} a$ unit speed time-like Legendre curve defined on an open interval $I_{2}$ containing 0 , and $p=p(t)$ a complex-valued function defined on $I_{2}$. Then the Lagrangian immersion $L_{f p z}:\left(U_{f z}, g_{f p z}\right) \rightarrow \mathbf{C}_{1}^{2}$ defined by

$$
L_{f p z}(s, t)=f(s) z(t)-\int_{0}^{t} p(t) z^{\prime}(t) d t
$$

is minimal if and only if either $L_{f p z}$ is a totally geodesic Lagrangian surface or, up to reparametrization and rigid motions, $L_{f p z}$ is an open portion of the Lagrangian minimal surface $M_{1}$ defined by (6.2). 
B.-Y. CHEN

Proof. Let $f: I_{1} \rightarrow \mathbf{C}^{*}$ be a unit speed curve, $z: I_{2} \rightarrow S_{2}^{3}(1) \subset \mathbf{C}_{1}^{2}$ a unit speed time-like Legendre curve defined on an open interval $I_{2}$ containing 0 , and $p=p(t)$ a complex-valued function defined on $I_{2}$. Then it follows from (4.5), (6.1), Proposition 3.1 and Theorem 4.1 that $L_{f p z}$ is a minimal Lagrangian immersion if and only if the following two conditions hold identically:

(i) The curvature function $\kappa(s)$ of $f$ in $\mathbf{C}$ satisfies

$$
\kappa(s)|f(s)-p(t)|^{2}+\left\langle f^{\prime}(s), i f(s)-i p(t)\right\rangle=0 .
$$

(ii) The curvature function $\lambda(t)$ of the Legendre curve $z(t)$ in $S_{2}^{3}(1)$ satisfies

$$
\lambda(t)|f(s)-p(t)|^{2}+\left\langle p^{\prime}(t), i f(s)-i p(t)\right\rangle=0 .
$$

Let us assume that $L_{f p z}$ is a minimal Lagrangian immersion. In order to solve the differential system (6.7) and (6.8), we divide into two cases.

CASE $(\mathrm{a}): f^{\prime \prime}(s)=0$. In this case, we obtain that $\kappa=0$ and $f(s)=c_{1} s+c_{2}$ for some complex numbers $c_{1}, c_{2}$ with $\left|c_{1}\right|=1$. Moreover, it follows from (6.7) that $\left\langle f^{\prime}, i f-i p\right\rangle=0$. Thus, after applying (4.5) and (6.8), we conclude that $L_{f p z}$ is a totally geodesic Lagrangian immersion.

CASE (b): $f^{\prime \prime}(s) \neq 0$. In this case, we have $\kappa \neq 0$ because we have $f^{\prime \prime}=i \kappa f^{\prime}$. Now, by differentiating (6.7) with respect to $s$ we find

$$
\kappa^{\prime}(s)|f(s)-p(t)|^{2}+3 \kappa\left\langle f^{\prime}(s), f(s)-p(t)\right\rangle=0,
$$

which can be expressed as

$$
(\ln |\kappa(s)|)^{\prime}+\frac{3}{2} \frac{\partial}{\partial s}\left(\ln |f-p|^{2}\right)=0 .
$$

By solving (6.9) we get

$$
|f-p|^{2}=\frac{\psi^{2}(t)}{\kappa^{2 / 3}(s)}
$$

for some real-valued function $\psi(t)$. Substituting (6.10) into (6.7) gives

$$
\left\langle f(s)-p(t), i f^{\prime}(s)\right\rangle=\psi^{2}(t) \kappa^{1 / 3}(s) .
$$

Differentiating (6.11) with respect to $s$ gives

$$
\left\langle f(s)-p(t), f^{\prime}(s)\right\rangle=-\frac{\kappa^{\prime} \psi^{2}(t)}{3 \kappa^{5 / 3}} .
$$

Since $f$ is a unit speed curve with $\kappa \neq 0, f^{\prime}(s), f^{\prime \prime}(s) / \kappa$ form an orthonormal frame over $\mathbf{R}$. Thus, we obtain from (6.11) and (6.12) that

$$
f(s)=p(t)-\kappa^{1 / 3}\left(\frac{\kappa^{\prime}}{3 \kappa^{2}}-i\right) \psi^{2}(t) f^{\prime}(s) .
$$

By differentiating (6.13) with respect to $t$, we obtain

$$
p^{\prime}(t)=2 \kappa^{1 / 3}\left(\frac{\kappa^{\prime}}{3 \kappa^{2}}-i\right) \psi(t) \psi^{\prime}(t) f^{\prime}(s)
$$

If $\psi^{\prime}(t) \neq 0$, then $(6.14)$ gives

$$
\frac{p^{\prime}(t)}{\psi(t) \psi^{\prime}(t)}=2 \kappa^{1 / 3}\left(\frac{\kappa^{\prime}}{3 \kappa^{2}}-i\right) f^{\prime}(s),
$$


which implies that

$$
p^{\prime}(t)=2 \delta \psi(t) \psi^{\prime}(t), \quad\left(\frac{\kappa^{\prime}}{3 \kappa^{5 / 3}}-i \kappa^{1 / 3}\right) f^{\prime}(s)=\delta
$$

for some complex number $\delta$. Thus, we have

$$
p(t)=\psi^{2}(t) \delta+\eta
$$

for some complex numbers $\delta, \eta$. Substituting (6.17) into (6.13) gives

$$
f(s)=\left\{\delta-\left(\frac{\kappa^{\prime}}{3 \kappa^{5 / 3}}-i \kappa^{1 / 3}\right) f^{\prime}(s)\right\} \psi^{2}(t)+\eta,
$$

which implies that $\psi$ is constant. Thus $p$ is also constant by $(6.17)$. Hence, we conclude from (6.8) that $\lambda(t)=0$. Consequently, $z=z(t)$ is a Legendre geodesic in $S_{2}^{3}(1) \subset \mathbf{C}_{1}^{2}$. Moreover, we find from (6.10) that

$$
|f(s)-p|^{2}=\left(\frac{a^{2}}{\kappa}\right)^{2 / 3}
$$

where $p \in \mathbf{C}$ and $0 \neq a \in \mathbf{R}$. Substituting (6.19) into (6.7) gives

$$
\left\langle i f^{\prime}(s), f(s)-p\right\rangle=a^{4 / 3} \kappa^{1 / 3} \text {. }
$$

Now, let us reparametrize $f$ as $f(s(x))=p+x+i y(x)$. Then we have

$$
\kappa=\frac{y^{\prime \prime}(x)}{\left(1+y^{\prime}(x)^{2}\right)^{3 / 2}}, \quad\left\langle i f^{\prime}, f-p\right\rangle=\frac{y-x y^{\prime}(x)}{\left(1+y^{\prime}(x)^{2}\right)^{1 / 2}} .
$$

By combining (6.20) and (6.21), we get

$$
a^{4} y^{\prime \prime}(x)=\left(y-x y^{\prime}(x)\right)^{3} .
$$

Let $u=y-x y^{\prime}$. We get $d u / d x=-x y^{\prime \prime}$. Thus, (6.22) becomes $a^{4} u^{\prime}(x)=-x u^{3}$. Solving this equation gives

$$
y-x y^{\prime}= \pm \frac{a^{2}}{\sqrt{x^{2}-a^{4} c}}
$$

for some constant $c$. After solving this differential equation, we have

$$
y=\mp\left(\frac{\sqrt{x^{2}-a^{4} c}}{a^{2} c}+b x\right)
$$

for some real number $b$. Thus, we find

$$
f=p+(1 \mp i b) x \mp i \frac{\sqrt{x^{2}-a^{4} c}}{a^{2} c} .
$$

From (6.21) and (6.23), we discover that

$$
\kappa=\frac{ \pm a^{8} c^{3}}{\left(\left(x+a^{2} b c \sqrt{x^{2}-a^{4} c}\right)^{2}+a^{4} c^{2}\left(x^{2}-a^{4} c\right)\right)^{3 / 2}} .
$$

Hence, we obtain from (6.19), (6.24) and (6.25) that

$$
\left(x+a^{2} b c \sqrt{x^{2}-a^{4} c}\right)^{2}+a^{4} c^{2}\left(x^{2}-a^{4} c\right)=a^{4} c^{2}\left(x^{2}+\left(\frac{\sqrt{x^{2}-a^{4} c}}{a^{2} c}+b x\right)^{2}\right)
$$

which implies that

$$
b^{2}=\frac{1-a^{4} c^{2}}{a^{4} c^{2}}
$$


Thus $a^{4} c^{2} \leq 1$. Consequently, we see from (6.23) that $f(s)-p=x(s)+i y(s)$ lies in the hyperbola:

$$
x^{2} \pm \frac{2 \sqrt{1-a^{4} c^{2}}}{a^{2} c} x y-y^{2}=\frac{1}{c}
$$

Furthermore, it follows from (6.6) that

$$
L_{f p z}=(f(s)-p) z(t)=(x(s)+i y(s)) z(t) .
$$

By applying the following rotation:

$$
\begin{aligned}
& x=\cos \left(\frac{1}{2} \tan ^{-1}\left(\frac{\sqrt{1-a^{4} c^{2}}}{a^{2} c}\right)\right) \tilde{x} \mp \sin \left(\frac{1}{2} \tan ^{-1}\left(\frac{\sqrt{1-a^{4} c^{2}}}{a^{2} c}\right)\right) \tilde{y} \\
& y= \pm \sin \left(\frac{1}{2} \tan ^{-1}\left(\frac{\sqrt{1-a^{4} c^{2}}}{a^{2} c}\right)\right) \tilde{x}+\cos \left(\frac{1}{2} \tan ^{-1}\left(\frac{\sqrt{1-a^{4} c^{2}}}{a^{2} c}\right)\right) \tilde{y}
\end{aligned}
$$

on $\mathbf{C}^{2}$, equation (6.25) becomes $\tilde{x}^{2}-\tilde{y}^{2}=a^{2}$. Thus may assume that

$$
f(s)-p=a\left(\cosh \left(\frac{s}{a}\right)+i \sinh \left(\frac{s}{a}\right)\right) \text {. }
$$

Since $z(t)$ is a unit speed Legendre geodesic in $S_{2}^{3}(1) \subset \mathbf{C}_{1}^{2}$ which can be represented by

$$
z(t)=(\sinh t, \cosh t)
$$

Consequently, up to reparametrization and rigid motions, $L_{f p z}$ is an open portion of the Lagrangian minimal surface $M_{0}$ defined by (6.2).

The converse can be verified by direct computation.

Theorem 6.2. Let $f: I_{1} \rightarrow \mathbf{C}^{*}$ be a unit speed curve, $z: I_{2} \rightarrow H_{1}^{3}(-1) \subset \mathbf{C}_{1}^{2}$ a unit speed space-like Legendre curve defined on an open interval $I_{2}$ containing 0 , and let $p=p(t)$ be a complex-valued function defined on $I_{2}$. Then the Lagrangian immersion $L_{f p z}:\left(U_{f z}, g_{f p z}\right) \rightarrow \mathbf{C}_{1}^{2}$ defined by

$$
P_{f p z}(s, t)=f(s) z(t)-\int_{0}^{t} p(t) z^{\prime}(t) d t
$$

is minimal if and only if either $P_{f p z}$ is a totally geodesic Lagrangian surface or, up to reparametrization and rigid motions, $P_{f p z}$ is an open portion of the Lagrangian minimal surface defined by (6.4).

Proof. Let $f: I_{1} \rightarrow \mathbf{C}^{*}$ be a unit speed curve, $z: I_{2} \rightarrow H_{1}^{3}(-1) \subset \mathbf{C}_{1}^{2}$ a unit speed space-like Legendre curve defined on an open interval $I_{2}$ containing 0 , and let $p=p(t)$ be a complex-valued function defined on $I_{2}$. Then it follows from (4.11), (6.1), Theorem 3.2 and Theorem 4.2 that $P_{f p z}$ is minimal if and only if the following two conditions hold:

(i) The curvature function $\kappa(s)$ of $f$ in $\mathbf{C}$ satisfies

$$
\kappa(s)|f(s)-p(t)|^{2}+\left\langle f^{\prime}(s), i f(s)-i p(t)\right\rangle=0 .
$$

(ii) The curvature function $\lambda(t)$ of the Legendre curve $z(t)$ in $S_{2}^{3}(1)$ satisfies

$$
\lambda(t)|f(s)-p(t)|^{2}+\left\langle p^{\prime}(t), i p(t)-i f(s)\right\rangle=0 .
$$

Since these two conditions are exactly the corresponding two conditions given in the proof of Theorem 6.1. Therefore, we may obtain this theorem by using the same argument using in the proof of Theorem 6.1 . 


\section{REFERENCES}

[1] Aiyama, R., Lagrangian surfaces in the complex 2-space, in: Proceedings of the Fifth International Workshop on Differential Geometry (Taegu, 2000), 25-29, Kyungpook Natl. Univ., Taegu, 2001.

[2] Aiyama, R., Totally real surfaces in the complex 2-space, in: Steps in Differential Geometry (Debrecen, 2000), 15-22, Debrecen, 2001.

[3] Castro, I. and Chen, B.-Y., Lagrangian surfaces in complex Euclidean plane via spherical and hyperbolic curves, Tohoku Math. J. 58 (2006) 565-579.

[4] Castro, I. and Lerma, A. M., A new construction of Lagrangians in the complex Euclidean plane in terms of planar curves, J. Geom. Phys. 75 (2014), 162-172.

[5] Chen, B.-Y., Complex extensors and Lagrangian submanifolds in complex Euclidean spaces, Tohoku Math. J. 49 (1997), 277-297.

[6] Chen, B.-Y., Interaction of Legendre curves and Lagrangian submanifolds, Israel J. Math. 99 (1997), 69-108.

[7] Chen, B.-Y., Representation of flat Lagrangian $H$-umbilical submanifolds in complex Euclidean spaces, Tohoku Math. J. 51 (1999), 13-20.

[8] Chen, B.-Y., Complex extensors and Lagrangian submanifolds in indefinite complex Euclidean spaces, Bull. Inst. Math. Acad. Sinica 31 (2003), 151-179.

[9] Chen, B.-Y., Lagrangian surfaces of constant curvature in complex Euclidean plane, Tohoku Math J. 56 (2004), 289-298.

[10] Chen, B.-Y., Classification of Lagrangian surfaces of constant curvature in complex Euclidean plane, Proc. Edinburgh Math. Soc. 48 (2005), 337-364.

[11] Chen, B.-Y., Maslovian Lagrangian surfaces of constant curvature in complex projective or complex hyperbolic planes, Math. Nachr. 278 (2005), 1242-1281.

[12] Chen, B.-Y., Classification of Lagrangian surfaces of constant curvature in complex complex projective planes, J. Geom. Phys. 53 (2005), 428-460.

[13] Chen, B.-Y., Classification of Lagrangian surfaces of constant curvature in complex hyperbolic planes, J. Geom. Phys. 55 (2005), 399-439.

[14] Chen, B.-Y., Three additional families of Lagrangian surfaces of constant curvature in complex projective plane, J. Geom. Phys. 56 (2006), 666-669.

[15] Chen, B.-Y., Construction of Lagrangian surfaces in complex Euclidean plane with Legendre curves, Kodai Math. J. 29 (2006), 84-112.

[16] Chen, B.-Y., Classification of Lagrangian surfaces of constant curvature in complex hyperbolic planes, II, Soochow J. Math. 33 (2007), 127-165.

[17] Chen, B.-Y., Pseudo-Riemannian Geometry, $\delta$-invariants and Applications, World Scientific, Hackensack, NJ, 2011.

[18] Chen, B.-Y., Classification of spherical Lagrangian submanifolds in complex Euclidean spaces, Int. Electron. J. Geom. 6 (2013), no. 2, 1-8.

[19] Chen, B.-Y., Dillen, F., Verstraelen, L. and Vrancken, L., Lagrangian isometric immersions of a real-space-form $M^{n}(c)$ into a complex-space-form $\tilde{M}^{n}(4 c)$, Math. Proc. Cambridge Phil. Soc. 124 (1998), 107-125.

[20] Chen, B.-Y. and Ogiue, K., On totally real submanifolds, Trans. Amer. Math. Soc. 193 (1974), 257-266.

[21] Chen, B.-Y. and Vrancken,L., Lagrangian minimal isometric immersions of a Lorentzian real space form into a Lorentzian complex space form, Tohoku Math. J. 54 (2002), 121-143.

[22] Joyce, D., Special Lagrangian $m$-folds in $\mathbf{C}^{m}$ with symmetries, Duke Math. J. 115 (2002), $1-51$.

[23] Vrancken, L., Minimal Lagrangian submanifolds with constant sectional curvature in indefinite complex space forms, Proc. Amer. Math. Soc. 130 (2002), 1459-1466.

Department of Mathematics, Michigan State University, 619 Red Cedar Road, East Lansing, Michigan 48824-1027, U.S.A.

E-mail address: bychen@math.msu.edu 